\title{
VEGFA GENE variation influences hallucinations and frontotemporal morphology in psychotic disorders: a B-SNIP study
}

Paulo Lizano (1) 1,2, Olivia Lutz (1)', George Ling (1)', Jaya Padmanabhan (1) ${ }^{1,2}$, Neeraj Tandon', John Sweeney ${ }^{3}$, Carol Tamminga ${ }^{4}$, Godfrey Pearlson ${ }^{5}$, Gualberto Ruaño ${ }^{6}$, Mohan Kocherla ${ }^{6}$, Andreas Windemuth ${ }^{6}$, Brett Clementz ${ }^{7}$, Elliot Gershon (D) $^{8}$ and Matcheri Keshavan ${ }^{1,2}$

\begin{abstract}
Vascular endothelial growth factor A (VEGFA) dysfunction may contribute to a number of pathological processes that characterize psychotic disorders. However, the influence of VEGFA gene variants on clinical and neuroimaging phenotypes in psychotic disorders has yet to be shown. In the present study, we examined whether different VEGFA gene variants influence psychosis risk, symptom severity, cognition, and brain volume. The study group included 480 probands (Bipolar I disorder with psychosis, $n=205$; Schizoaffective disorder, $n=112$; Schizophrenia, $n=163$ ) and 126 healthy controls that were recruited across six sites in the B-SNIP consortium. VEGFA variants identified for analysis (rs699947, rs833070, and rs2146323) were quantified via SNP chip array. We assessed symptoms and cognition using standardized clinical and neuropsychological batteries. The dorsolateral prefrontal cortex (DLPFC), medial temporal lobe, and hippocampal volumes were quantified using FreeSurfer. In our sample, VEGFA rs2146323 A-carriers showed reduced odds of being a proband $(p=0.037, \mathrm{OR}=0.65,95 \% \mathrm{Cl}=0.43-0.98)$ compared to noncarriers, but not for rs699947 or rs833070. In probands, rs2146323 A- carriers demonstrated fewer hallucinations ( $p=0.035$, Cohen's $d=$ 0.194), as well as significantly greater DLPFC $(p<0.05$, Cohen's $d=-0.21)$ and parahippocampal volumes $(p<0.01$, Cohen's $d=-0.27$ ). No clinical or neuroimaging associations were identified for rs699947 or rs833070. In general, we found that the three SNPs exhibited several significant negative relationships between psychosis symptoms and brain structure. In the probands and control groups, positive relationships were identified between several cognitive and brain volume measures. The findings suggest VEGFA effects in the DLPFC and hippocampus found in animals may also extend to humans. VEGFA variations may have important implications in identifying dimensional moderators of function that could be targeted through VEGFA-mediated interventions.
\end{abstract}

\section{Introduction}

Vascular endothelial growth factor A (VEGFA) dysfunction may contribute to a number of pathological

\footnotetext{
Correspondence: Paulo Lizano (lizanopl@gmail.com)

'Department of Psychiatry, Beth Israel Deaconess Medical Center, Boston, MA, USA

${ }^{2}$ Department of Psychiatry, Harvard Medical School, Boston, MA, USA

Full list of author information is available at the end of the article.
}

processes that characterize psychotic disorders. VEGFA is an essential molecule for angiogenesis, neurogenesis, and brain plasticity and is distinct from other neurotrophic factors in its major angiogenic function ${ }^{1,2}$. VEGFA can promote proliferation of endothelial cells, neural stem cells, neurons, and mature astrocytes ${ }^{1}$. In the adult rodent brain, an enriched environment or overexpression of VEGFA can stimulate neovascularization, hippocampal 
neurogenesis, and improve cognitive performance, whereas blocking endogenous VEGFA function impairs the angiogenic, neurogenic, and cognitive response to neuro-enhancing stimuli ${ }^{1-6}$.

In schizophrenia, altered $V E G F A$ signaling has been implicated via transciptomic and epigenomic analysis of prefrontal cortical post mortem samples ${ }^{5,7-11}$. Imaging genetic studies of VEGFA single nucleotide polymorphisms (SNPs) in healthy subjects have demonstrated that minor allele carriers for rs833070 and rs2146323 have smaller hippocampal volumes and rs699947 minor allele carriers have reduced total gray and white matter volume $^{12,13}$. These polymorphisms were also significantly different $(p<3 \mathrm{e}-3, \mathrm{OR}=0.97$ to 1.04 , comparing schizophrenia vs. controls) in the Psychiatric Genome Consortium (PGC) SZ working group analysis, but this did not meet genome-wide level of significance at $5<10 \mathrm{e}-8^{14}$. It is well established that antipsychotic treatment results in changes in VEGFA levels that are related to the Positive and Negative Symptom Scale (PANSS) positive symptoms but not negative symptoms. Additionally, we have identified robust cognitive deficits and smaller medial temporal lobe (MTL) structures in psychosis probands compared to controls, and these abnormalities correlated with cognition and psychosis severity ${ }^{15-17}$. However, there are no studies to date examining the effects of these VEGFA polymorphisms on clinical and structural neuroimaging phenotypes in psychotic disorders.

This study was based on the cross-sectional BipolarSchizophrenia Network on Intermediate Phenotypes (BSNIP) consortium, which assessed clinical, cognitive, and neurobiological phenotypes across the psychotic spectrum. We took an integrated genetic and phenotype approach to (1) investigate the disease risk associated with rs699947, rs833070, and rs2146323, (2) determine the effects of these genetic variants on positive symptoms, memory, and executive function, and (3) evaluate their influence on DLPFC and MTL structure as well as hippocampal morphology.

\section{Materials and methods \\ Study participants}

The study group included 480 probands $(n=205$ Bipolar I with psychosis, BPP; $n=112$ Schizoaffective disorder, SAD; $n=163$ Schizophrenia, SZ) and 126 healthy controls $(\mathrm{HC})$ that were recruited as part of the BSNIP multisite study at Harvard University, Wayne State University, Maryland Psychiatric Research Center, University of Texas Southwestern Medical Center at Dallas, Institute of Living/Yale University, and University of Illinois at Chicago. Detailed sample information is provided elsewhere and participants provided informed consent ${ }^{18}$.

All subjects met the following inclusion criteria: ages 15-65, sufficient English proficiency, no history of neurological disorders or head injuries, no history of substance abuse in the last month or substance dependence in the last 6 months and a negative urine toxicology screen on the day of testing. Healthy controls also met criteria for no personal history of recurrent mood disorders, no personal or family history of psychotic disorders, no history of substance dependence and no history of any significant cluster A axis II personality features within the structured interview for DSM-IV-TR Personality. All sites used identical recruitment, clinical and diagnostic approaches that were approved by respective institutional review boards ${ }^{18}$.

Diagnoses were based on the Structured Clinical Interview for DSM-IV-TR (SCID-IV). Subjects received consensus diagnosis using information from clinical interviews, medical records, and family members. Symptom ratings were completed using PANSS. We focused our analysis on total positive symptom, delusion, and hallucination scores since they have been previously associated with $V E G F A^{10,19,20}$. Cognition was evaluated using the Brief Assessment of Cognition (BACS) battery and a full presentation of these data is available in ref. ${ }^{15}$. The Verbal Memory, Digit Sequence, Symbol Coding, and Tower of London subscales from BACS were used to assess verbal declarative memory, working memory, processing speed, and reasoning/problem solving, respectively. All scores were adjusted for age and sex and transformed to z-scores with a range of \pm 4.0 .

\section{Structural T1 imaging}

Structural T1-weighted images were acquired across six sites: Boston (3.0 T, GE Signa), Detroit (3.0 T, Siemens Allegra), Baltimore (3.0 T, Siemens Trio trim), Hartford (3.0 T, Siemens Allegra), Dallas (3.0 T, Philips), and Chicago (3.0 T, GE Signa). Structural T1 MPRAGE isovoxel scans $\left(\mathrm{TR}=6.7 \mathrm{~ms}, \mathrm{TE}=3.1 \mathrm{~ms}, 8^{\circ}\right.$ flip angle, $256 \times 240$ matrix size, total scan duration $=5: 26 \mathrm{~min}, 170$ sagittal slices, $1 \mathrm{~mm}$ slice thickness, $1 \times 1 \times 1.2 \mathrm{~mm}^{3}$ voxel resolution) were acquired with the Alzheimer's Disease Neuroimaging Initiative (ADNI) protocol (http://adni.loni.usc. edu).

Scans were assessed for motion and scanner artifacts and 51 subjects failed the first level of quality control. The passing scans were processed using FreeSurfer $5.1^{21}$ and were run through a first-level auto-reconstruction. Trained raters removed dura, sinuses, and vessels from the skull-stripped images that could interfere with segmentation and all raters demonstrated a 95\% inter-rater reliability. The images were run through a second- and third-level auto-reconstruction to extract volume measures. The gray matter volume for the dorsolateral prefrontal cortex (DLPFC) (calculated by summating the caudal and rostral middle frontal regions), entorhinal, 
parahippocampal, and hippocampus were extracted from the DKT atlas and bilateral values were summated.

\section{SNP selection and genotyping}

The human VEGFA gene is located on chromosome 6p12 (43,768,581-43,788,115) (GRCh38.p7; GCF_0000 01405.33) and includes eight exons separated by seven introns. We selected rs833070 (intron), rs2146323 (intron), and rs699947 (promoter) for tag SNPs that influence brain morphology in healthy individuals ${ }^{12,22}$, confer schizophrenia risk ${ }^{14}$, and have minor allele frequencies (MAF) $\geq 0.05$ in the HapMap-CEU population. More specifically, we examined publicly available databases, reviewed the literature on VEGFA polymorphisms in schizophrenia, and selected high-risk variants from NCBI, and the subsequent list was cross-referenced with the PGC schizophrenia working group findings which led to a selection of the three above-mentioned SNPs.

Blood samples were drawn after structural MRI scanning. Subjects' DNA was genotyped using an Illumina's Human Omni1-Quad Bead Chip for 1,140,419 common SNPs at Genomas Inc (Hartford Hospital, CT) and detailed information can be found at Meda et al. ${ }^{23}$. Genotyped data were preprocessed in PLINK v1.07 (http:// pngu.mgh.harvard.edu/purcell/plink/) following a published guideline for SNP selection in candidate genes/ regions $^{24}$. As an exploratory aim, we evaluated SNP distribution across biotypes generated by Clementz et al. using electrophysiology, eye tracking, and cognitive measures in the B-SNIP study with biotype 1 being the most compromised and 3 the least ${ }^{25}$.

\section{Statistical analyses}

A one-way analysis of variance and $\chi^{2}$ tests were used to test for between-group differences in demographic and clinical variables. We performed the analysis in the proband (combined groups consisting of SZ, SAD, BPP) vs. control groups to enhance the study power and to demonstrate that VEGFA genotype to phenotype features cross diagnostic boundaries. Genotype and allele frequencies were calculated by the gene-counting method and each polymorphism was tested for Hardy-Weinberg equilibrium using $X^{2}$ goodness-of-fit tests using the $\mathrm{R}$ package SNPassoc (http://www.creal.cat/jrgonzalez/ software.htm). The association between target SNPs and the risk of being a proband under two inheritance models, codominant and dominant models, was performed using logistic regression, adjusting for age, sex, and ethnicity factors. To validate our results, we performed a (1) sitespecific analysis with sites containing a large enough sample size and (2) we created a training and test dataset with a 70/30 random split of our whole sample. All statistical tests were two-sided and $p<0.05$ was defined as statistically significant. Bonferroni correction was applied by multiplying the $p$ values by the three SNPs being examined, which were carefully selected by identifying relevant SNPs from the PGC schizophrenia and bipolar datasets, as well as two structural neuroimaging studies $^{12-14,26}$. Subjects with imaging measures outside of four standard deviations were removed $(n=1)$ and those between three and four standard deviations were winsorized to the third standard deviation $(n=12)$. Contrasts between carriers and noncarriers within a group ( $\mathrm{HC}$ or Probands) were run on clinical, cognitive and imaging measures using an analysis of covariance (ANCOVA) or general linear hypothesis test while controlling for age, sex, ethnicity, and site for a total of 36 comparisons (3 SNPs and 12 traits) for a Bonferroni corrected $p$ value of 0.0014 . In probands, partial correlations within a phenotype and genotype were run using Kendall-tau correlations, adjusting for age, sex, ethnicity, and site.

As in previous genetic neuroimaging studies ${ }^{12,13,27}$ we included age, sex, ethnicity, and site as covariates when examining clinical and neuroimaging variables and excluded intracranial volume (ICV) for neuroimaging analysis in our basic model. We avoided correcting for ICV since it could be related to the disease and diminish the possibility of detecting associations that differentiate cases and controls, or that are related to the evolution of the disease. We also performed an analysis covarying for duration of illness, PANSS total negative symptoms, and average chlorpromazine equivalents. Also, the genotypes were dichotomized into those homozygous for the more frequent allele and "carriers" of the less frequent allele.

\section{Code availability}

Code is available upon request my emailing the corresponding author.

\section{Results}

Our VEGFA SNP sample included 606 participants (HC, $n=126$, Proband, $n=480$ ), consisting of 303 males (HC, $n=53$; Proband, $n=250$ ) and 303 females (HC, $n=$ 73; Proband, $n=230$ ) (Supplementary Table S1). The mean age of our sample was 35.6 years $(\mathrm{HC}, 37.3$ years \pm 12.4; Proband, 35.2 years \pm 12.5 ). The groups did not differ by age $(\mathrm{F}=2.7 ; p=0.10)$, sex $(\mathrm{F}=3.6 ; p=0.057)$ or ethnicity $(\mathrm{F}=3.3 ; p=0.192)$. However, they did show significant site $(\mathrm{F}=13.1 ; p=0.022)$ effects but no group by site interactions were observed. Sample characteristics by diagnostic group or biotype group, and polymorphism can be found in Supplementary Tables S2, S3.

\section{Genotype distribution}

The VEGFA polymorphisms were in Hardy-Weinberg equilibrium in probands, $\mathrm{HC}$, and in the combined group $(p>1 \mathrm{e}-5)$. The call rates were $98.5 \%$ for rs $2146323,99.9 \%$ for rs 833070 , and $92.5 \%$ for rs699947 in the two groups. 
Table 1 Logistic regression analysis of VEGFA SNPs in probands and control subjects

\begin{tabular}{|c|c|c|c|c|c|c|c|c|c|c|c|c|c|c|}
\hline & \multicolumn{7}{|c|}{ Genotype } & \multicolumn{7}{|l|}{ Allele } \\
\hline & $N(\%)$ & & & OR & $95 \% \mathrm{Cl}$ & $p$ value & Adj $p$ value & $N(\%)$ & & OR & $95 \% \mathrm{Cl}$ & $p$ value & Adj $p$ value & $p$ of $\mathrm{HWE}^{\mathrm{a}}$ \\
\hline rs699947 & GG & GT & $\pi$ & & & & & GG & $\mathrm{T}-$ & & & & & \\
\hline \multirow[t]{2}{*}{ Controls } & $40(35)$ & $49(43)$ & $26(22)$ & 0.89 & $0.56-1.43$ & & & $40(35)$ & $75(65)$ & & & & & 0.15 \\
\hline & 169 (38) & $185(41)$ & $92(21)$ & 0.84 & $0.48-1.46$ & 0.80 & 0.79 & $169(38)$ & $277(62)$ & 0.87 & $0.57-1.34$ & 0.54 & 0.56 & \\
\hline \multicolumn{15}{|l|}{ Probands } \\
\hline rs833070 & GG & $A G$ & AA & & & & & GG & A- & & & & & \\
\hline \multirow[t]{2}{*}{ Controls } & $37(29)$ & $64(51)$ & $25(20)$ & 0.67 & $0.43-1.05$ & & & $37(29)$ & $89(71)$ & & & & & 0.78 \\
\hline & $176(37)$ & $204(42)$ & $99(21)$ & 0.83 & $0.47-1.46$ & 0.21 & 0.23 & $176(37)$ & $303(63)$ & 0.72 & $0.47-1.10$ & 0.12 & 0.12 & \\
\hline \multicolumn{15}{|l|}{ Probands } \\
\hline rs2146323 & $C C$ & $A C$ & AA & & & & & $C C$ & A- & & & & & \\
\hline \multirow[t]{2}{*}{ Controls } & $47(40)$ & $64(54)$ & $7(6)$ & 0.56 & $0.37-0.85$ & & & $47(40)$ & $71(60)$ & & & & & 0.01 \\
\hline & $242(51)$ & $184(38)$ & $53(11)$ & 1.47 & $0.63-3.43$ & $0.005^{*}$ & $0.007^{*}$ & $242(51)$ & $237(49)$ & 0.65 & $0.43-0.98$ & 0.037 & 0.042 & \\
\hline Probands & & & & & & & & & & & & & & \\
\hline
\end{tabular}

VEGFA vascular endothelial growth factor A, SNP single nucleotide polymorphism, $N$ number, OR odds ratio, $C l$ confidence interval, $H W E$ Hardy-Weinberg Equilibrium *Survived Bonferroni correction; Adj $p$ value covaried for age, sex, race

${ }^{a} H W E$ test was carried out in controls

The bolded values stand for signficant uncorreted $p$ values $(p<0.05)$

Table 2 Effect sizes for rs699947, rs833070, and rs2146323 SNP effect on psychopathology and cognition in probands and control subjects

\begin{tabular}{|c|c|c|c|c|c|c|c|c|c|c|c|c|}
\hline \multirow[b]{3}{*}{ Psychopathology } & \multicolumn{6}{|l|}{ NC } & \multicolumn{6}{|c|}{ Probands } \\
\hline & \multicolumn{2}{|c|}{ rs699947 (GG vs. T-) } & \multicolumn{2}{|c|}{ rs833070 (GG vs. A-) } & \multicolumn{2}{|c|}{ rs2146323 (CC vs. A-) } & \multicolumn{2}{|c|}{ rs699947 (GG vs. T-) } & \multicolumn{2}{|c|}{ rs833070 (GG vs. A-) } & \multicolumn{2}{|c|}{ rs2146323 (CC vs. A- } \\
\hline & $\begin{array}{l}p \text { value } \\
-\end{array}$ & $\begin{array}{l}\text { Cohen's } d \\
-\end{array}$ & $\begin{array}{l}p \text { value } \\
-\end{array}$ & $\begin{array}{l}\text { Cohen's } d \\
-\end{array}$ & $\begin{array}{l}p \text { value } \\
-\end{array}$ & $\begin{array}{l}\text { Cohen's } d \\
-\end{array}$ & $\begin{array}{l}p \text { value } \\
0.873\end{array}$ & $\begin{array}{l}\text { Cohen's } d \\
0.015\end{array}$ & $\begin{array}{l}p \text { value } \\
0.728\end{array}$ & $\begin{array}{l}\text { Cohen's d } \\
0.032\end{array}$ & $\begin{array}{l}p \text { value } \\
0.396\end{array}$ & $\begin{array}{l}\text { Cohen's } d \\
0.079\end{array}$ \\
\hline Delusions & - & - & - & - & - & - & 0.615 & -0.049 & 0.649 & -0.043 & 0.931 & 0.009 \\
\hline Hallucinations & - & - & - & - & - & - & 0.109 & 0.157 & 0.059 & 0.176 & 0.035 & 0.194 \\
\hline Cognition & $p$ value & Cohen's $d$ & $p$ value & Cohen's $d$ & $p$ value & Cohen's $d$ & $p$ value & Cohen's $d$ & $p$ value & Cohen's $d$ & $p$ value & Cohen's $d$ \\
\hline Composite & 0.210 & -0.224 & 0.427 & -0.146 & 0.198 & -0.249 & 0.727 & -0.033 & 0.675 & -0.038 & 0.559 & -0.053 \\
\hline Verbal Memory & 0.998 & 0.020 & 0.578 & -0.106 & 0.234 & -0.219 & 0.072 & -0.167 & 0.140 & -0.135 & 0.23 & -0.109 \\
\hline Digit Sequence & 0.213 & -0.218 & 0.453 & -0.146 & 0.108 & -0.299 & 1.000 & -0.007 & 0.904 & -0.011 & 0.994 & 0.000 \\
\hline Tower of London & 0.093 & -0.326 & 0.371 & -0.164 & 0.298 & -0.221 & 0.284 & -0.103 & 0.599 & -0.049 & 0.741 & -0.030 \\
\hline Symbol Coding & 0.565 & -0.109 & 0.468 & -0.129 & 0.150 & -0.269 & 0.637 & 0.046 & 0.649 & -0.039 & 0.519 & -0.059 \\
\hline
\end{tabular}

Psychopathology, PANSS (Positive and Negative Syndrome Scale); Cognition, BACS (Brief Assessment of Cognition); for psychopathology measures covaried for age, sex, site, and ethnicity. Adjusted cognition measures were covaried for site and ethnicity

$\mathrm{HC}$ healthy control

The bolded values stand for signficant uncorreted $p$ values $(p<0.05)$

The analysis for the three tag SNPs revealed a significant difference for rs2146323 in the distribution of the codominant $(p=0.005, \mathrm{OR}=1.47,95 \% \mathrm{CI}=0.63-3.43)$ and dominant $(p=0.037, \mathrm{OR}=0.65,95 \% \mathrm{CI}=0.43-0.98)$ models between probands and HC (Table 1). The significant association between rs2146323 and proband membership survived adjustment for age, sex, and ethnicity in the codominant $(p=0.007)$ and dominant model $(p=0.042)$. For rs2146323, the A- carriers had significantly lower odds of being a proband when compared to the $\mathrm{CC}$ individuals. No significant association was observed between the other two tag SNPs and proband membership $(p>0.05)$. We replicated our finding for rs2146323 in our site-specific analysis at our Baltimore site ( $\mathrm{HC} n=37$, Proband $n=130$, codominant $p=0.002$ ), but not for the other three sites. We found rs833070 to be significantly different in our Detroit site (HC $n=20$, Proband $n=49$, codominant $p=0.039$ ), but not in the other sites. We replicated our rs2146323 finding in the training set ( $\mathrm{HC} n=88$, Proband $n=336$, codominant $p$ $=0.0143)$, but not in the test dataset $(\mathrm{HC} n=38$, Proband $n=144$, codominant $p=0.513$ ). Biotype 1 group was significantly less likely to be an A-carrier compared to the 


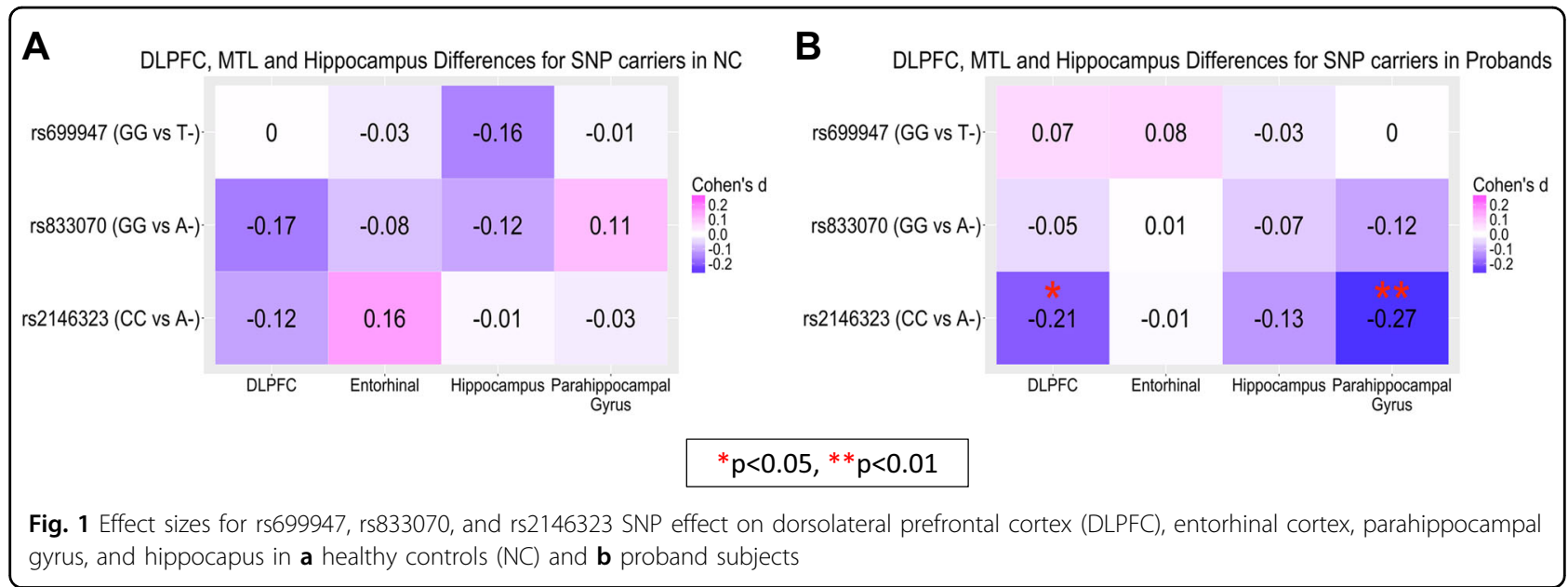

other biotype or diagnostic groups (Supplementary Table S3).

\section{Psychopathology and cognition}

The PANSS scores for the probands were: positive total score $=15.2 \pm 5.5$; delusions score $=2.5 \pm 1.4$; and hallucinations score $=2.4 \pm 1.5$ (Supplementary Table S1). There were no significant associations between rs699947 or rs833070 and PANSS positive total, delusions, or hallucinations scores (Table 2). For rs2146323 we found that, compared with subjects homozygous for the $\mathrm{C}$ allele, Acarriers had significantly reduced PANSS hallucinations scores $(p=0.035$, Cohen's $d=-0.194)$. There was no significant difference between scores for positive total or delusions and rs2146323 A- carriers (Table 2). The results were no longer significant after adding average daily chlorpromazine equivalence as a covariate.

The proband and control groups differed by Verbal Memory $(\mathrm{F}=56 ; p=<0.001)$, Digit Sequence $(\mathrm{F}=67 ; p$ $=<0.001)$, Tower of London $(\mathrm{F}=19 ; p=<0.001)$, and Symbol Coding ( $\mathrm{F}=120 ; p=<0.001)$ (Supplementary Table S1). There were no SNP effects on cognition in either the control or proband group (Table 2).

\section{Neuroimaging data}

No significant differences for ICV $(\mathrm{F}=0.94, p=0.33)$ existed between probands and HC (Supplementary Table S1). There were significant smaller volumes in probands compared to HC for the DLPFC, hippocampus, parahippocampus, and entorhinal cortex (data not shown).

In controls, there were no significant findings identified between rs699947, rs833070, or rs2146323 and brain structure (Fig. 1). Also, there weren't any significant differences found between brain structure and rs699947 or rs833070 in the proband group. However, for rs2146323 in probands we found that, compared with subjects homozygous for the $\mathrm{C}$ allele, A- carriers demonstrated greater brain volume in the DLPFC $(p<0.05$, Cohen's $d=$ $-0.21)$ and parahippocampal gyrus $(p<0.01$, Cohen's $d=$ -0.27 ) when covaried for age, sex, ethnicity, and site, but not for the entorhinal cortex or hippocampus (Fig. 1). Both regions remain significant after adding total PANSS negative symptoms as a covariate (DLPFC, $p=0.038$, Cohen's $d=-0.211$; parahippocampal gyrus, $p=0.007$, Cohen's $d=-0.277$ ) but was no longer significant after covarying for total ICV $(p>0.05)$. Parahippocampal volume remained significant after adding duration of illness $(p=0.005$, Cohen's $d=-0.287)$ and average daily chlorpromazine equivalence $(p=0.001$, Cohen's $d=$ $-0.414)$ as covariates.

\section{Clinical and BACS correlations in probands}

In probands, homozygotes for the major allele for each of the three SNPs displayed significant negative correlations $(p<0.05)$ between PANSS positive symptoms and DLPFC and hippocampal volumes, with fewer relationships observed for rs833070, and no relationship was observed between PANSS positive symptoms and entorhinal or parahippocampal volumes (Fig. 2). For cognition in probands, homozygotes for the major allele for each of the three SNPs displayed significant $(p<0.05)$ positive relationships between cognitive measures and hippocampal volume with rs246323 also having additional significant parahippocampal correlations (Fig. 2). Many of the same significant positive relationships were observed between cognition and hippocampal volume in the control sample with the addition of several significant positive correlations with the DLPFC (Fig. 3).

Similar to the above findings, minor allele carriers for each of the three SNPs showed significant negative relationships between PANSS positive symptoms and DLPFC and hippocampal volume (Fig. 2). Interestingly, rs2146323 A- carriers did not demonstrate a significant relationship between hallucination severity and hippocampal or 


\begin{tabular}{|c|c|c|c|c|c|c|c|c|c|c|c|c|}
\hline \multirow[b]{2}{*}{$\begin{array}{l}\text { PANSS } \\
\text { Positive Total }\end{array}$} & \multicolumn{4}{|c|}{ rs699947 GG Individuals } & \multicolumn{4}{|c|}{ rs833070 GG Individuals } & \multicolumn{4}{|c|}{ rs2146323 CC Individuals } \\
\hline & $\stackrel{* *}{*}+19$ & 0 & $\begin{array}{l}\star \star * \\
-0.17\end{array}$ & -0.06 & $\stackrel{\star \star \star}{*}-17$ & -0.04 & -0.1 & -0.04 & $\begin{array}{l}\star \star \star \star \\
-0.17\end{array}$ & -0.05 & -0.15 & -0.04 \\
\hline Delusions- & $\begin{array}{l}* * * \\
-0.21\end{array}$ & -0.07 & -0.16 & -0.05 & $\begin{array}{l}\star \star \star \star \\
-0.2\end{array}$ & -0.08 & -0.12 & -0.05 & $\begin{array}{l}* \star \star * \\
-0.18\end{array}$ & -0.03 & -0.13 & -0.04 \\
\hline Hallucinations & -0.15 & -0.03 & -0.13 & -0.06 & $\begin{array}{l}* * \\
-0.15\end{array}$ & -0.05 & -0.07 & -0.07 & -0.14 & -0.07 & -0.12 & -0.07 \\
\hline $\begin{array}{l}\text { Verbal. } \\
\text { Memory }\end{array}$ & 0.04 & 0 & 0.12 & 0.1 & 0.03 & 0.01 & 0.12 & 0.11 & 0.03 & 0.04 & 0.11 & 0.09 \\
\hline $\begin{array}{l}\text { Tower of } \\
\text { London }\end{array}$ & 0.09 & 0.02 & 0.11 & 0.08 & 0.07 & 0.03 & 0.13 & 0.05 & 0.07 & 0.04 & 0.13 & 0.09 \\
\hline $\begin{array}{l}\text { Symbol } \\
\text { Coding }\end{array}$ & -0.03 & 0.01 & 0.14 & 0.08 & 0 & 0.01 & 0.14 & 0.07 & 0 & 0.01 & 0.13 & 0.09 \\
\hline $\begin{array}{r}\text { Digit } \\
\text { Sequencing }\end{array}$ & 0.1 & 0.01 & 0.19 & 0.14 & 0.08 & 0.03 & $\begin{array}{l}* \star * \star \\
0.21\end{array}$ & 0.16 & 0.08 & 0.02 & $\begin{array}{l}* * * \\
0.19\end{array}$ & $\begin{array}{l}* * * \\
0.16\end{array}$ \\
\hline \multirow[t]{2}{*}{$\begin{array}{r}\text { BACS } \\
\text { Composite }\end{array}$} & 0.07 & 0.01 & 0.15 & 0.11 & 0.05 & 0 & 0.17 & 0.11 & 0.04 & 0.01 & 0.16 & 0.12 \\
\hline & \multicolumn{4}{|c|}{ rs699947 T- Carriers Gyrus } & \multicolumn{2}{|c|}{ rs833070 A- Carriers } & $\begin{array}{l}\text { Hippocampus } \\
\text { A- Carriers }\end{array}$ & $\begin{array}{l}\text { Parahippocampal } \\
\text { Gyrus }\end{array}$ & \multicolumn{3}{|c|}{ rs2146323 A- Carriers } & $\begin{array}{l}\text { Parahippocampal } \\
\text { Gyrus }\end{array}$ \\
\hline $\begin{array}{l}\text { PANSS } \\
\text { Positive Total }\end{array}$ & -0.1 & -0.04 & -0.13 & -0.08 & -0.11 & -0.02 & $\begin{array}{l}\star \star \star \\
-0.17\end{array}$ & -0.08 & -0.1 & -0.02 & $\stackrel{* *}{*}$ & -0.09 \\
\hline Delusions & $\begin{array}{l}\star \star \star \star \\
-0.16\end{array}$ & -0.06 & $\stackrel{\star * *}{-0.12}$ & -0.09 & $\begin{array}{l}\star \star \star \star \\
-0.15\end{array}$ & -0.02 & $\begin{array}{l}* \star \star \\
-0.13\end{array}$ & -0.09 & $\begin{array}{l}\star \star \star \star \\
-0.16\end{array}$ & -0.07 & $\begin{array}{l}\star \star * \\
-0.13\end{array}$ & -0.11 \\
\hline Hallucinations. & -0.08 & -0.03 & -0.09 & -0.07 & -0.08 & -0.01 & $\stackrel{* * *}{* * 11}$ & -0.05 & -0.09 & 0.01 & -0.07 & -0.06 \\
\hline $\begin{array}{l}\text { Verbal } \\
\text { Memory }\end{array}$ & 0.09 & 0.07 & 0.1 & 0.07 & 0.08 & 0.08 & 0.1 & 0.07 & 0.07 & 0.05 & 0.1 & 0.06 \\
\hline $\begin{array}{l}\text { Tower of } \\
\text { London }\end{array}$ & 0.13 & 0.04 & 0.13 & 0.11 & $\begin{array}{l}* * * \\
0.16\end{array}$ & 0.04 & 0.11 & 0.1 & 0.16 & 0 & 0.09 & 0.04 \\
\hline $\begin{array}{l}\text { Symbol } \\
\text { Coding }\end{array}$ & 0.08 & 0 & 0.13 & 0.1 & 0.08 & 0 & 0.1 & 0.1 & 0.07 & 0.01 & 0.1 & 0.08 \\
\hline $\begin{array}{r}\text { Digit. } \\
\text { Sequencing }\end{array}$ & 0.04 & -0.02 & 0.1 & 0.09 & 0.06 & -0.02 & 0.09 & 0.09 & 0.03 & 0 & 0.08 & 0.06 \\
\hline \multirow[t]{2}{*}{$\begin{array}{r}\text { BACS } \\
\text { Composite }\end{array}$} & 0.11 & 0.03 & $\begin{array}{l}\star \star \star * \\
0.17\end{array}$ & 0.12 & 0.13 & 0.05 & 0.14 & 0.12 & $0^{*}$ & 0.07 & 0.13 & 0.09 \\
\hline & $\begin{array}{l}\text { DLPFC } \\
\text { rrelation } \\
\text { efficient } \\
.2 \\
.1 \\
.0 \\
0.1 \\
0.2\end{array}$ & Entorhinal & Hippocampus & $\begin{array}{l}\text { Parahippocampal } \\
\text { Gyrus }\end{array}$ & DLPFC & Entorhinal & Hippocampus & $\begin{array}{l}\text { Parahippocampal } \\
\text { Gyrus }\end{array}$ & DLPFC & Entorhinal & Hippocampus & $\begin{array}{l}\text { Parahippocampal } \\
\text { Gyrus }\end{array}$ \\
\hline
\end{tabular}

Fig. 2 DLPFC, entorhinal, parahippocampal, and hippocampal volume correlations with symptom severity and cognition in probands by rs699947, rs833070, and rs 2146323 SNP status. Values in the box indicate Kendall-tau correlations. ${ }^{*} p<0.05 ;{ }^{* *} p<0.01 ;{ }^{* * *} p<0.001$ (Survived Bonferroni correction)

DLPFC volume. Additionally, in probands we found that minor allele carriers for each of the three SNPs still demonstrated a significant positive relationship between cognitive domains and hippocampal volume, except for rs2146323. In controls, there were no relationships between cognition and hippocampal volume in minor allele carriers for each of the three SNPs tested, except for rs2146323, which showed significant positive relationships between symbol coding or BACS composite score and hippocampal volume (Fig. 3).

\section{Discussion}

In summary, we found that rs2146323 A- carriers compared to $\mathrm{CC}$ individuals had significantly reduced odds of being a proband and that the biotype 1 group (most neurobiologically compromised) were less likely to be A- carriers. This finding was replicated in one of our sites and in our training dataset. No significant effect was noted for rs699947 or rs833070. We also demonstrated that rs2146323 A- carriers in probands were associated with reduced hallucinations and greater volume in the DLPFC and parahippocampus. We did not identify any significant clinical, cognitive, or structural associations for rs699947 or rs833070 in probands or controls. Finally, we showed that all three SNPs exhibited several significant negative relationships between psychosis symptoms and brain structure, as well as showing that in probands and control groups, positive relationships were identified between several cognitive and brain volume measures. These results support our hypothesis that VEGFA polymorphisms may not only play a role in reducing psychosis odds, but that rs2146323 might confer a neuroprotective effect on clinical and neuroimaging phenotypes.

In this cross-sectional study, we found that rs 2146323 A- carriers were associated with a reduced odd of being a psychosis proband and we were able to replicate this finding, while rs833070 or rs699947 were not risk conferring. The PGC SZ working group identified a nongenome-wide significant associated risk for rs699947, rs833070, and rs2146323 ${ }^{14}$, which further enhances the significance of our finding. However, the PGC bipolar disorder working group did not identify an associated risk for rs699947 or rs2146323 (rs833070 was not included), which included a mix of bipolar patients that were not differentiated for lifetime psychotic symptoms ${ }^{26}$. These discrepant results might be due to our inclusion of psychotic bipolar I disorder subjects, which is distinct from the bipolar types used in the PGC analysis. Finally, a cross-sectional genetic study of schizophrenia in a Han Chinese population showed that rs699947 nominally decreased the risk of schizophrenia in a recessive inheritance model, but they did not identify an association for 


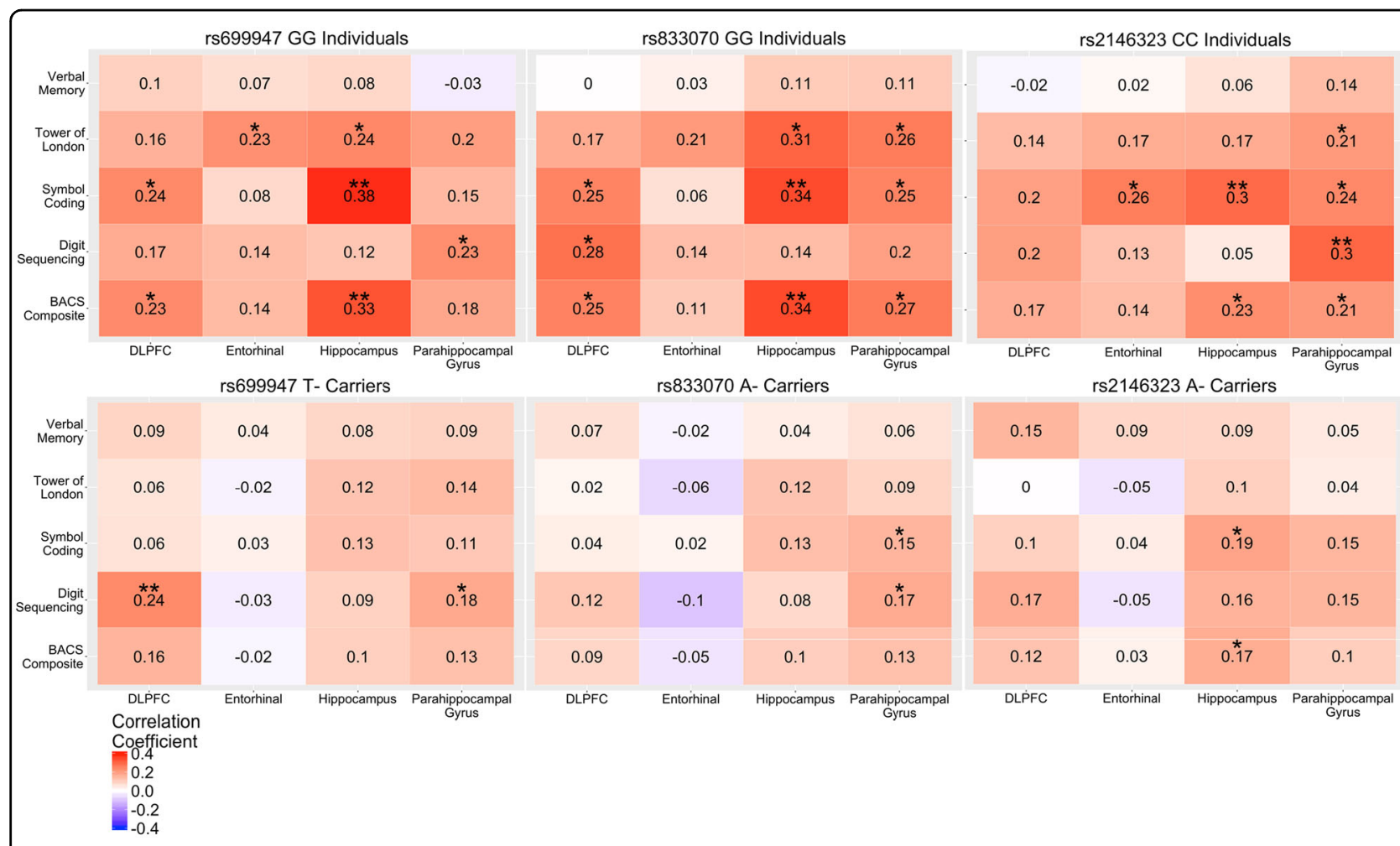

Fig. 3 DLPFC, entorhinal, parahippocampal, and hippocampal volume correlations with cognition in healthy controls by rs699947, rs833070, and rs2146323 SNP status. Values in the box indicate Kendall-tau correlations. ${ }^{*} p<0.05 ;{ }^{* *} p<0.01 ;{ }^{* * *} p<0.001$ (Survived Bonferroni correction)

rs833070, and they didn't test for rs $2146323^{22}$. To the best of our knowledge we are the first group to demonstrate that the VEGFA polymorphism, rs2146323, has a dimensional effect on psychosis membership.

The function of rs 2146323 has yet to be determined, but we do know that it lies within intron 2, is located in 111bp $5^{\prime}$ of exon 3 , is distant from predicted transcription factor binding sites, and is not predicted to be involved in exon splicing alterations ${ }^{28}$. Only one study has examined the effect of the rs2146323 variant on peripheral VEGFA levels and they identified a significant association in their detection sample, but not in their replication sample ${ }^{29}$. Other studies have implicated it as a predictive maker for treatment response in improving lung function in asthmatic patients ${ }^{30}$, enhancing anatomic outcome in agerelated macular degeneration (ARMD) ${ }^{31}$, and increased retinal thickness in $\mathrm{ARMD}^{32}$. In regards to rs699947 and rs833070, they are located on the promoter region of $V E G F A$, can result in changes in VEGF expression levels, and have been proposed to have a pharmacogenetic association in neovascular ARMD, but with inconsistent findings ${ }^{32}$. Our finding that $\mathrm{rs} 2146323$ is less frequently present in our most compromised biotype 1 group further suggests a biologically plausible mechanism involving $V E G F A$. It is possible that rs2146323 and/or rs699947 might alter VEGFA expression or isoform formation, which could influence brain development, neuroplasticity, and neuro-protection. Thus, these observations make rs2146323 a promising target for unveiling the mechanism of this polymorphism on VEGFA expression, structure, and function.

In this study, we demonstrate that rs2146323 A- carriers exert a specific reduction in PANSS hallucination subscale scores but no effect was identified for rs833070 or rs699947. There are no human or animal studies evaluating the effects of VEGFA polymorphisms on psychotic or cognitive phenotypes in psychotic disorders. However, our findings align with animal studies, which have shown that VEGFA, VEGF receptor 1 (VEGFR1/Flt-1), and VEGF receptor 2 (VEGFR2/KDR) signaling affect working memory primarily by modulating neuroplasticity at the hippocampus or through peripheral neurotrophic effects $^{1-6,33}$. Our findings are further supported by human studies, which have demonstrated that the effects of psychotic symptoms are mixed ${ }^{34}$, whereas VEGR1/Flt-1 and VEGFR2/KDR dysregulation were associated with worse psychotic symptoms in a prospective familial high risk for psychosis study ${ }^{35,36}$, a post mortem SZ study ${ }^{10}$, and in case reports of systemic VEGFR1/Flt-1 administration $^{19,20,37}$. Additionally, treatment with antipsychotic or antidepressant medications has been shown to modulate VEGF levels and is associated with improved 
treatment outcomes in SZ, bipolar and major depressive disorder $^{38-41}$. Also, we would hypothesize that since rs2146323 is a potential pharmacogenetic predictor of treatment response, that the association between fewer hallucinations and rs2146323 carrier status could reflect treatment response to antipsychotics.

In our neuroimaging analysis, we found that the neuroprotective effects of rs 2146323 on clinical phenotypes were also evident across the hypothesized brain regions associated with psychosis. Specifically, we found that rs2146323 A- carriers exhibited greater volume in the dorsolateral prefrontal and parahippocampal regions in probands, and this effect was specific to rs2146323 and not rs699947 or rs833070. This is the first study to research the effects of polymorphisms on brain structure in a psychotic disorder group. One healthy volunteer study assessed the effects of four variants (rs833068, rs833070, rs2146323, and rs3025020), and showed that rs2146323 and rs833070 carriers had reduced hippocampal concentrations compared to major allele carriers, but their sample was smaller than ours ${ }^{12}$. Additionally, we observed in probands that rs699947 T- or rs2146323 Acarriers have inverse relationships between PANSS positive symptoms and prefrontal-hippocampal structure, which is an effect also seen in major allele carriers for those same SNPs. In regards to cognition, we found that the positive relationship between BACS composite score and hippocampal volume persisted for rs2146323 Acarriers and CC individuals in the proband and control group, which suggests that more brain volume was related to better cognition and lower symptom levels. Taken together, our findings relate to animal and human studies showing that it modulates cytoarchitecture, hallucinations, and memory in psychotic disorders.

The findings suggest that the effects of prefrontal cortex and hippocampus found in animals extend to humans and further understanding of the effects of variation might have important implications for identifying a subgroup of patients with psychosis that are more vulnerable to DLPFC and hippocampal pathology, as well as those that are most likely to benefit from mediating interventions.

\footnotetext{
Author details

${ }^{1}$ Department of Psychiatry, Beth Israel Deaconess Medical Center, Boston, MA, USA. ${ }^{2}$ Department of Psychiatry, Harvard Medical School, Boston, MA, USA. ${ }^{3}$ Department of Psychiatry, University of Cincinnati Medical Center, Cincinnati, $\mathrm{OH}$, USA. ${ }^{4}$ University of Texas Southwestern Medical Center, Dallas, TX, USA. ${ }^{5}$ Hartford Hospital, Yale School of Medicine, Hartford, CT, USA. ${ }^{6}$ Genomas Inc. Hartford, CT, USA. ${ }^{7}$ Department of Psychology, University of Georgia, Athens, GA, USA. ${ }^{8}$ Department of Psychiatry and Behavioral Neurosciences, University of Chicago, Chicago, IL, USA
}

Conflict of interest

The authors declare that they have no conflict of interest.
Publisher's note

Springer Nature remains neutral with regard to jurisdictional claims in published maps and institutional affiliations.

Supplementary Information accompanies this paper at (https://doi.org/ 10.1038/s41398-018-0271-y).

Received: 27 August 2018 Accepted: 10 September 2018

Published online: 11 October 2018

\section{References}

1. Jin, K. et al. Vascular endothelial growth factor (VEGF) stimulates neurogenesis in vitro and in vivo. Proc. Natl Acad. Sci. USA 99, 11946-11950 (2002).

2. Fabel, $K$. et al. VEGF is necessary for exercise-induced adult hippocampal neurogenesis. Eur. J. Neurosci. 18, 2803-2812 (2003).

3. Cao, L. et al. VEGF links hippocampal activity with neurogenesis, learning and memory. Nat. Genet. 36, 827-835 (2004).

4. During, M. J. \& Cao, L. VEGF, a mediator of the effect of experience on hippocampal neurogenesis. Curr. Alzheimer Res. 3, 29-33 (2006).

5. Howell, K. R., Kutiyanawalla, A. \& Pillai, A. Long-term continuous corticosterone treatment decreases VEGF receptor-2 expression in frontal cortex. PLOS ONE 6, e20198 (2011).

6. Kiuchi, T., Lee, H. \& Mikami, T. Regular exercise cures depression-like behavior via VEGF-Flk-1 signaling in chronically stressed mice. Neuroscience 207, 208-217 (2012).

7. Fulzele, S. \& Pillai, A. Decreased VEGF mRNA expression in the dorsolateral prefrontal cortex of schizophrenia subjects. Schizophr. Res. 115, 372-373 (2009).

8. Maycox, P. R. et al. Analysis of gene expression in two large schizophrenia cohorts identifies multiple changes associated with nerve terminal function. Mol. Psychiatry 14, 1083-1094 (2009).

9. Harris, L. W. et al. Comparison of peripheral and central schizophrenia biomarker profiles. PLOS ONE 7, e46368 (2012)

10. Hino, M. et al. Decreased VEGFR2 expression and increased phosphorylated Akt1 in the prefrontal cortex of individuals with schizophrenia. J. Psychiatr. Res. 82, 100-108 (2016).

11. Lee, S. A. \& Huang, K. C. Epigenetic profiling of human brain differential DNA methylation networks in schizophrenia. Bmc Med. Genom. 9(Suppl 3), 68 (2016).

12. Blumberg, H. P. et al. Influence of vascular endothelial growth factor variation on human hippocampus morphology. Biol. Psychiatry 64, 901-903 (2008).

13. Takeuchi, $H$. et al. The VEGF gene polymorphism impacts brain volume and arterial blood volume. Hum Brain Mapp 38, 3516-3526 (2017).

14. Schizophrenia Working Group of the Psychiatric Genomics C. Biological insights from 108 schizophrenia-associated genetic loci. Nature 511, 421-427 (2014).

15. Hill, S. K. et al. Neuropsychological impairments in schizophrenia and psychotic bipolar disorder: findings from the Bipolar-Schizophrenia Network on Intermediate Phenotypes (B-SNIP) study. Am. J. Psychiatry 170, 1275-1284 (2013).

16. Mathew, I. et al. Medial temporal lobe structures and hippocampal subfields in psychotic disorders: findings from the Bipolar-Schizophrenia Network on Intermediate Phenotypes (B-SNIP) study. JAMA Psychiatry 71, 769-777 (2014).

17. Padmanabhan, J. L. et al. Correlations between brain structure and symptom dimensions of psychosis in schizophrenia, schizoaffective, and psychotic bipolar I disorders. Schizophr. Bull. 41, 154-162 (2015).

18. Tamminga, C. A. et al. Clinical phenotypes of psychosis in the BipolarSchizophrenia Network on Intermediate Phenotypes (B-SNIP). Am. J. Psychiatry 170, 1263-1274 (2013).

19. Kunene, V. \& Porfiri, E. Sunitinib-induced acute psychosis: case report. Clin. Genitourin. Cancer 9, 70-72 (2011).

20. Kuo, Y. L., Yang, Y. K., Cheng, H. C., Yen, C. J. \& Chen, P. S. Psychotic disorder induced by a combination of sorafenib and BAY86-9766. Gen. Hosp. Psychiatry 36, 450 e5-e7 (2014).

21. Desikan, R. S. et al. An automated labeling system for subdividing the human cerebral cortex on MRI scans into gyral based regions of interest. Neuroimage 31, 968-980 (2006).

22. Gao, K et al. Association study of VEGFA polymorphisms with schizophrenia in Han Chinese population. Neurosci. Lett. 590, 121-125 (2015). 
23. Meda, S. A. et al. Multivariate analysis reveals genetic associations of the resting default mode network in psychotic bipolar disorder and schizophrenia. Proc. Natl Acad. Sci. USA 111, E2066-E2075 (2014).

24. Pettersson, F. H. et al. Marker selection for genetic case-control association studies. Nat. Protoc. 4, 743-752 (2009).

25. Clementz, B. A. et al. Identification of distinct psychosis biotypes using brainbased biomarkers. Am. J. Psychiatry 173, 373-384 (2016).

26. Psychiatric GCBDWG. Large-scale genome-wide association analysis of bipolar disorder identifies a new susceptibility locus near ODZ4. Nat. Genet. 43, 977-983 (2011).

27. Kalmady, S. V. et al. Relationship between Interleukin-6 gene polymorphism and hippocampal volume in antipsychotic-naive schizophrenia: evidence for differential susceptibility? PLOS ONE 9, e96021 (2014).

28. Churchill, A. J. et al. VEGF polymorphisms are associated with severity of diabetic retinopathy. Invest. Ophthalmol. Vis. Sci. 49, 3611-3616 (2008).

29. Ruggiero, D. et al. Genetics of VEGF serum variation in human isolated populations of cilento: importance of VEGF polymorphisms. PLOS ONE 6, e16982 (2011)

30. Sharma, S. et al. Association of VEGF polymorphisms with childhood asthma, lung function and airway responsiveness. Eur. Respir. J. 33, 1287-1294 (2009).

31. Immonen, I. et al. Vascular endothelial growth factor gene variation and the response to photodynamic therapy in age-related macular degeneration. Ophthalmology 117, 103-108 (2010).

32. Hagstrom, S. A. et al. VEGFA and VEGFR2 gene polymorphisms and response to anti-vascular endothelial growth factor therapy: comparison of age-related macular degeneration treatments trials (CATT). JAMA Ophthalmol. 132 521-527 (2014).
33. Licht, T. et al. Reversible modulations of neuronal plasticity by VEGF. Proc. Nat/ Acad. Sci. USA 108, 5081-5086 (2011).

34. Pillai, A. et al. Association of serum VEGF levels with prefrontal cortex volume in schizophrenia. Mol. Psychiatry 21, 686-692 (2016).

35. Lizano, P. L. et al. Angiogenic and immune signatures in plasma of young relatives at familial high-risk for psychosis and first-episode patients: a preliminary study. Schizophr. Res. 170, 115-122 (2016).

36. Lizano, P. et al. Association of sFlt-1 and worsening psychopathology in relatives at high risk for psychosis: a longitudinal study. Schizophrenia Res $\mathbf{1 8 3}$ 75-81 (2016).

37. Demirci, N. S., Erdem, G. U., Dogan, M., Ozdemir, N. Y. \& Zengin, N. A rare case: hallucination associated with pazopanib. J. Cancer Res. Ther. 11, 961-962 (2015).

38. Kim, T. H., Kdh, Lee, S. K., Son, B. K. \& Jung, J. S. The effect of antipsychotic drug treatment on serum VEGF, sVEGFR-1, and sVEGFR-2 level in schizophrenia-a preliminary study. Korean J. Biol. Psychiatry 14, 232-240 (2007).

39. Haring, L. et al. Antipsychotic treatment reduces psychotic symptoms and markers of low-grade inflammation in first episode psychosis patients, but increases their body mass index. Schizophr. Res. 169, 22-29 (2015).

40. Benedetti, F. et al. Stem Cell Factor (SCF) is a putative biomarker of antidepressant response. J. Neuroimmune Pharmacol. 11, 248-258 (2016).

41. Warner-Schmidt, J. L. \& Duman, R. S. VEGF is an essential mediator of the neurogenic and behavioral actions of antidepressants. Proc. Natl Acad. Sci. USA 104, 4647-4652 (2007). 